HIAS-E-59

\title{
The Effect of Democratic Decision Making on Investment in Reputation
}

\author{
Ruth Ben-Yashar \\ Department of Economics, BarIlan University, Ramat Gan 52900, Israel \\ Miriam Krausz \\ School of Economics, Ashkelon Academic College, 12 Yitzhak Ben-Tzvi, Ashkelon, Israel \\ Shmuel Nitzan \\ Department of Economics, BarIlan University, Ramat Gan 52900, Iseael \\ Hitotsubashi Institute for Advanced Study, Hitotsubashi University
}

November 2017

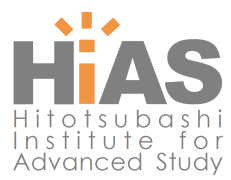

Hitotsubashi Institute for Advanced Study, Hitotsubashi University 2-1, Naka, Kunitachi, Tokyo 186-8601, Japan

tel:+81 425808604 http://hias.ad.hit-u.ac.jp/

HIAS discussion papers can be downloaded without charge from: http://hdl.handle.net/10086/27202

https://ideas.repec.org/s/hit/hiasdp.html

All rights reserved. 


\title{
The effect of democratic decision making on investment in reputation
}

\author{
by
}

\author{
Ruth Ben-Yashar ${ }^{\text {a }}$, Miriam Krausz ${ }^{\mathrm{b}}$ and Shmuel Nitzan ${ }^{\text {c.d }}$
}

Students wish to increase the probability of being admitted to a prestigious school. Job candidates are interested in the probability of getting a desirable job. Defendants are concerned about the probability of being acquitted. In all such binary settings, the probability of the desirable outcome to individuals can be affected by their reputation. Applying a standard uncertain dichotomous choice benchmark setting, we focus on how the nature of the applied decision-making rule affects the individuals' incentive to invest in improvement of their reputation. Our main results establish that a democratic (non - democratic) decision-making system based on the simple majority (unanimity) rule ensures maximal (minimal) marginal productivity of reputation that increases (decreases) with the size of the decision-making committee.

Keywords: decision making structure, investment in reputation.

Classification Codes: D7,

${ }^{a}$ Department of Economics, Bar Ilan University, Ramat Gan 52900, Israel.

${ }^{\mathrm{b}}$ School of Economics, Ashkelon Academic College, 12 Yitzhak Ben-Tzvi, Ashkelon, Israel.

c Department of Economics, Bar Ilan University, Ramat Gan 52900, Israe and Hitotsubashi Institute for Advanced Study, Hitotsubashi University.

${ }^{d}$ The third author is grateful to HIAS for enabling the completion of this paper. 


\section{Introduction}

Group binary decision making is very significant in Economics, Medicine, Law and other disciplines. Examples include juries deciding whether to acquit or convict a defendant, committees considering job candidates, projects or loans, boards of managers choosing between two policies and medical experts deciding whether to carry out a certain mode of treatment or procedure. In all of these settings the concerned individual may have an incentive to invest resources in increasing the probability of receiving a favorable outcome from the decision process. The defendant wishes to be acquitted, the job candidate would like to be selected, the entrepreneur would like to be approved for a loan, and so forth. These individuals are aware that certain environmental attributes enhance their reputation that positively affects the outcome of the decision process. For example, the neighborhood in which an individual grew up or lives in may be associated with high income, low crime and impressive scholastic achievements. The school that an individual attended may be known to produce successful graduates. In this paper we explore the role played by the type of decision-making system faced by the individual in determining his incentive to invest in reputation, focusing on two types of rules: the democratic simple majority rule and the non-democratic unanimity rule.

For ease of exposition, we have chosen to focus on the case of a defendant whose verdict is determined by a jury facing two states of nature (the defendant being guilty or innocent) that have some given a-priory probability and two possible decisions (conviction or acquittal). There are therefore four possible final decisions, two correct decisions (conviction of guilty and acquittal of innocent defendants) and two incorrect ones (acquittal of guilty and conviction of innocent defendants). The jury (committee) consists of several members (decision-makers) with the same preferences who seek to maximize the probability to reach the correct decision. The final decision is reached by the jury decision rule. Most of the studies in this framework focus on the probability that the committee will choose the correct decision. ${ }^{1}$ In this paper we focus on the defendant's point of view vis. the probability

\footnotetext{
1 These studies follow Condorcet's (1785) approach. The Condorcet Jury Theorem defines the contributions under which the majority rule is an efficient aggregation of all the information from all the decision-makers. Various attempts have been made to generalize the theorem, for example, Nitzan and Paroush (1982), Grofman et al. (1983), Shapley and Grofman (1984), Ben-Yashar and Nitzan
} 
of his acquittal.

The probability of acquittal can be affected by the relevant evidence which is a major criterion for making a decision, as well as the number and skills of the committee members, the applied decision rule and the defendant's reputation. As noted, reputation consists of environmental factors such as the defendant's background, socio-economic status, residence neighborhood, education etc. and is general information. In a fair trial we would prefer the jury not to be biased by such information. We would like the jury to make a decision based on information pertaining purely to the crime for which the defendant is on trial. Nonetheless, defendants wish to increase the probability of being acquitted. They cannot change the evidence and they cannot control the size and the quality of the committee. However, they can invest in their reputation, which consists of the environmental factors, in order to affect the a-priory probability of innocence. ${ }^{2}$ The novelty of our model is that we analyze the effect of the defendant's reputation on the outcome of the decision-making process

We focus on reputation, assuming that it is represented in the decision-making model by the a-priory probability of innocence. The question we ask is how the nature of the decision-making rule used by the court affects the incentive to invest in reputation. Our results clarify how the decision-making rule applied by the jury and the size of the decision-making group affects the benefit of reputation - the marginal increase in the probability of receiving an acquittal. ${ }^{3}$

More specifically, assuming that the a-priory probability of innocence is equal to one half, we show that the probability of a decision that is favorable for the defendant is equal to one half under the simple majority rule, regardless of the size and the quality of the decision-making committee. From the defendant's point of view,

(1997), Berend and Paroush (1998), Ben- Yashar and Danziger (2011) and Dietrich and List (2013). Discussion of extreme decision rules can be found in Sah and Stiglitz (1986) and Ben-Yashar and Nitzan (2001).

\footnotetext{
${ }^{2}$ Some papers discuss the significance of the prior on a correct decision in committees, see for example Ben-Yashar and Nitzan (2014).

${ }^{3}$ In the literature on strategic deliberation, the optimal amount of information acquisition is discussed in Persico (2004). Gerardi and Yariv (2008), show that the incentive to acquire information depends on the committee's decision rule. Also discussed is the effect of payoffs to decision makers' on the committee's behavior (e.g., Levy, 2007 and Midjord, et al. 2017).
} 
this is tantamount to tossing a coin. Under the unanimity rule, we show that the defendant is in a worse position. In this case, even though the committee has no bias in favor or against the defendant, still, his probability of acquittal is lower than one half. We explore the possibility of the defendant affecting his a-priory probability of innocence, i.e., investing in enhancing his reputation with the purpose of increasing the probability of a favorable decision. Since this process is costly for the individual, the individual's incentive to invest in reputation crucially depends on the marginal productivity of his investment. Marginal productivity is defined as the marginal increase in the probability of receiving a desired outcome from the jury. Our results establish that a decision-making system based on the democratic simple majority rule ensures maximal marginal productivity of reputation. It therefore yields the largest incentive to invest in reputation ${ }^{4}$. Moreover, the marginal productivity of investing in reputation increases with the size of the committee. Conversely, the decision-making system based on the non-democratic unanimity rule results in minimal productivity of reputation which decreases with the size of the committee.

\section{The model}

An $n=2 k+1$ member jury decides whether to acquit (1) or convict (-1) a defendant. The defendant is either innocent (1) or guilty (-1). The final decision is based on the individual jurors' decisions. There are therefore two possible correct decisions (1/1) (acquitting an innocent defendant), and (-1/-1) (convicting a guilty defendant) and two corresponding incorrect ones $((-1 / 1)$ and $(1 /-1))$. Let $\alpha$ be the a-priori probability that a defendant is innocent. If $\alpha=1 / 2$, the probabilities that defendants are guilty or innocent are equal. Let $x_{i}$ denote the juror $i$ 's decision, $x_{i}=1$ and $x_{i}=-1$ then represent acquittal and conviction, respectively. The vector $x=\left(x_{1}, \ldots, x_{n}\right)$ describes the decision profile of $n$ jurors making decisions simultaneously. Each juror chooses the correct alternative with probability $0.5<p<1,1$ if the defendant is innocent (1), and -1 if the defendant is guilty $(-1)$. Note that $(1-p)$ is the probability that the juror chooses the incorrect alternative: -1 if the defendant is innocent (1) and 1 if the defendant is guilty (-1). The final decision regarding the defendant is made using a decision rule $f$,

4 Reputation has an important role in decisions concerning individuals in a variety of settings. For example, reputation incentives and the return to reputation in a career model in Bar-Isaac and Deb (2014). The value of reputation information is discussed in Bolton, Ockenfels and Ebeling (2011). Seminal reputation models include Kreps and Wilson (1982) Bayesian updating model in which the "reputation effect" is demonstrated. 
whereby the function $f$ assigns 1 or -1 (acquittal or conviction) to a decision profile $x$ in $\{-1,1\}^{n}$. That is, $f:\{-1,1\}^{n} \rightarrow\{-1,1\}$. Supposing a qualified majority rule is used, $f=\left\{\begin{array}{ll}1 & N(1) \geq q \\ -1 & \text { otherwise }\end{array}\right\}$, where, $N(1)$ is the number of jurors who decide to acquit the defendant, $N(-1)$ is the number of jurors who decide to convict the defendant, $n$ is the total number of jurors and an integer $q$ is the quota required of jurors deciding to acquit that will lead to a verdict of acquittal. Hereafter q represents the decision rule 5 . Note that $q=k+1$ represents the simple majority rule and $q=n$ represents the unanimity rule, whereby only a unanimous verdict will lead to acquittal.

\section{Results}

In this section we examine the probability of a jury acquitting the defendant, and the effect of changes in the a-priory probability of innocence on the probability of acquittal. Let us denote the probabilities that an $n$-member jury acquits an innocent or guilty defendant by $T(1: 1)$, and $T(1:-1)$, respectively. Hence, the probability to acquit a defendant is denoted by $\operatorname{Pr}(1: q)$ where $\operatorname{Pr}(1: q)=\alpha T(1: 1)+(1-\alpha) T(1:-1)$.

Formally,

$$
\begin{aligned}
& T(1: 1)=\sum_{j=q}^{n}\left(\begin{array}{l}
n \\
j
\end{array}\right) p^{j}(1-p)^{n-j}, \text { and } T(1:-1)=\sum_{j=q}^{n}\left(\begin{array}{c}
n \\
j
\end{array}\right)(1-p)^{j} p^{n-j} . \text { Hence, } \\
& \frac{\partial \operatorname{Pr}(1: q)}{\partial \alpha}=T(1: 1)-T(1:-1)=\sum_{j=q}^{n} \Delta_{j}
\end{aligned}
$$

where $\Delta_{j}=\left(\begin{array}{c}n \\ j\end{array}\right)\left\{p^{j}(1-p)^{n-j}-(1-p)^{j} p^{n-j}\right\}$.

Since
(a) $\forall j>\frac{n}{2}, \Delta_{j}>0 .^{6}$
(b) $\forall j=a, \Delta_{a}=-\Delta_{n-a} \cdot 7$

\footnotetext{
${ }^{5}$ Using qualified majority rules is a plausible assumption in a model that assumes decision makers with homogenous skills (see Ben Yashar and Nitzan, 1997).

$6 \Delta_{j}>0 \Leftrightarrow\left(\frac{p}{1-p}\right)^{j}>\left(\frac{p}{1-p}\right)^{n-j} \Leftrightarrow j>n-j \Leftrightarrow j>n / 2$. (Note that under the model's assumptions, $\mathrm{p}>1 / 2$ and hence $\left.p / 1-p^{p 1}\right)$.
} 
If $q>\frac{n}{2}$, then by (a), $\frac{\partial \operatorname{Pr}(1: q)}{\partial \alpha}>0$. If $q<\frac{n}{2}$, then by (b), we know that, $\sum_{j=q}^{n} \Delta_{j}=\sum_{j=n-q+1}^{n} \Delta_{j}$. Since $n-q+1>n / 2$, this last term is positive by (a) and therefore, $\frac{\partial \operatorname{Pr}(1: q)}{\partial \alpha}>0$.

Hence, the probability to acquit a defendant increases with the a-priory probability that he is innocent.

We now focus on two types of decision rules. The democratic simple majority rule and the non-democratic unanimity rule. The following theorems show that the decision rule used to aggregate the decisions of the committee members is of crucial importance in determining the magnitude of the marginal effect of the a-priory probability of innocence on the probability to acquit.

\section{Theorem 1:}

Under the simple majority rule:

(a) Given equal a-priory probabilities that a defendant is guilty or innocent, there is no bias in favor of acquittal or conviction, i,e,. $\operatorname{Pr}(1: k+1)=\frac{1}{2}$, regardless of the size of the committee and the skills of its members.

(b) An increase in the a-priory probability of innocence produces the largest increase in the probability to acquit, compared to all other qualified decision rules.

(c) The increase in the probability to acquit resulting from an increase in the apriory probability of innocence, increases with the size of the committee.

Proof:

(a) According to the simple majority rule, $q=k+1$

$$
f=\left\{\begin{array}{ll}
1 & N(1) \geq k+1 \\
-1 & \text { otherwise }
\end{array}\right\}=\left\{\begin{array}{ll}
1 & N(-1) \leq k \\
-1 & \text { otherwise }
\end{array}\right\}=\left\{\begin{array}{ll}
1 & \text { otherwise } \\
-1 & N(-1) \geq k+1
\end{array}\right\}
$$

Hence $T(1: 1)=T(-1:-1)$ and $T(1:-1)=1-T(-1:-1)$, which implies that

${ }^{7} \Delta_{a}=\left(\begin{array}{l}n \\ a\end{array}\right)\left[p^{a}(1-p)^{n-a}-(1-p)^{a} p^{n-a}\right]$ and $\Delta_{n-a}=\left(\begin{array}{c}n \\ n-a\end{array}\right)\left[p^{n-a}(1-p)^{a}-(1-p)^{n-a} p^{a}\right]$. Hence, $\Delta_{a}=-\Delta_{n-a}$. 


$$
\operatorname{Pr}(1: k+1)=\frac{1}{2}(T(1: 1)+(1-T(-1:-1)))=\frac{1}{2} .
$$

(b) See Appendix A.

(c) $\frac{\partial \operatorname{Pr}(1: k+1)}{\partial \alpha}=2 \mathrm{~T}(1: 1)-1$. Based on the Condorcet Jury Theorem

$$
\frac{\partial T(1: 1)}{\partial n}>0, \text { hence, } \frac{\frac{\partial \operatorname{Pr}(1: k+1)}{\partial \alpha}}{\partial n}>0 .
$$

According to Theorem 1, when facing a committee that uses the simple majority rule, the defendant can expect that given equal a-priory probabilities that he is guilty or innocent, the probability of acquittal under the simple majority rule is $1 / 2$, regardless of whether the defendant is in fact innocent. Moreover, under these conditions the probability to acquit a defendant is independent of the number of jury members and their skills. This can be considered a desirable state from the jury's point of view, because it implies that there is no bias in favor or against the defendant- the decision is made solely on the basis of the merit of the relevant criteria. However, as shown, under the simple majority rule the defendant can expect to be acquitted or convicted with equal probability, the equivalent of tossing a coin. Should the defendant decide to increase the a-priory probability of innocence by investing in reputation, he can expect the largest marginal increase in the probability of being acquitted. That is, the simple majority rule induces the maximal increase in the probability to acquit a defendant in response to a marginal change in the a-priory probability that the defendant is innocent. It therefore induces the strongest incentive to invest in reputation. Finally, the marginal productivity of reputation increases with the size of the committee.

We now turn to case of the non-democratic unanimity rule to investigate the defendant's incentive to invest in reputation when the jury applies this rule.

\section{Theorem 2:}

Under the unanimity rule: 
(a) Given equal a-priory probabilities that a defendant is guilty or innocent, there is an implicit bias against acquittal, i.e., $\operatorname{Pr}(1: n)<\frac{1}{2}$, which depends on the size of the committee.

(b) An increase in the a-priory probability of innocence produces the smallest increase in the probability to acquit, compared to all qualified decision rules.

(c) The increase in the probability to acquit resulting from an increase in the apriory probability of innocence decreases with the size of the committee.

Proof:

(a)

$$
f=\left\{\begin{array}{ll}
1 & N(1) \geq q \\
-1 & \text { otherwise }
\end{array}\right\}=\left\{\begin{array}{ll}
1 & n-N(-1) \geq q \\
-1 & \text { otherwise }
\end{array}\right\}=\left\{\begin{array}{ll}
1 & \text { otherwise } \\
-1 & N(-1)>n-q
\end{array}\right\}
$$

If $q>k+1(n-q<k+1)$ then $T(1: 1)<T(-1:-1)$. Thus,

$\operatorname{Pr}(1: n)=\frac{1}{2}(T(1: 1)+T(1:-1))=\frac{1}{2}(T(1: 1)+1-T(-1:-1))$, which is less than $1 / 2$.

(b)See Appendix A.

(c) Under the unanimity rule, $q=n$,

$$
\begin{aligned}
& \frac{\partial \operatorname{Pr}(1: n)}{\partial \alpha}=T(1: 1)-T(1:-1)=p^{n}-(1-p)^{n} . \\
& \text { Hence } \frac{\frac{\partial \operatorname{Pr}(1: n)}{\partial \alpha}}{\partial n}=p^{n+1}-(1-p)^{n+1}-p^{n}+(1-p)^{n} .
\end{aligned}
$$

Since $p^{n}(p-1)<(1-p)^{n}(-1+1-p) \equiv\left(\frac{p}{1-p}\right)^{n}>\frac{p}{1-p} \equiv\left(\frac{p}{1-p}\right)^{n-1}>1$,

$$
\frac{\frac{\partial \operatorname{Pr}(1: n)}{\partial \alpha}}{\partial n}<0
$$

Q.E.D

Theorem 2 states that given equal a-priory probabilities that a defendant is guilty or innocent, when the decision rule is the non-democratic unanimity rule, the probability of acquittal is less than $1 / 2$, and is dependent on the number of jurors and on the probability each juror chooses the correct alternative. From the defendant's point of view, the probability of being acquitted is extremely low. Should the defendant wish to invest in reputation, thereby increasing the a-priory probability of 
innocence, he can expect the smallest increase in the probability of being acquitted under the unanimity rule in comparison to all other qualified decision rules. The marginal increase in the probability to be acquitted in response to an increase in the apriory probability of innocence, decreases with the size of the jury. Hence, defendants facing a jury that bases its decisions on the unanimity rule have the lowest incentive to invest in reputation when the jury is large.

\section{Conclusions}

Our note examines the impact of the democratic nature of the decision-making rule on the incentive to invest in reputation. Such investment increases the a-priory probability of innocence and, in turn, the probability of the acquittal decision. Since investment in reputation is costly, the effectiveness of such an investment is economically significant. Our results imply that the democratic nature of the decisionmaking rule determines both the incentive to invest in reputation as well as the effect of the size of the committee on this incentive. The largest incentive to invest in reputation exists in a large jury that applies the simple majority rule. On the other hand, the defendant will have to invest the largest amount of resources in reputation in order to achieve a target increase in the probability of gaining a favorable decision when facing a large jury that applies the extreme non-democratic decision rule.

To our knowledge, our model is the first to include in a collective-decision model the issue of the incentive of the agent affected by the collective decision to invest in reputation under alternative decision-making systems. Our results are applicable to all settings of binary decision making where the concerned individuals can influence the collective decision by investing in their reputation. These results give defendants and candidates a strategic decision tool for planning the investment of personal resources in reputation. In our setting, it is the combination of the type of the applied collective decision rule, democratic or non-democratic, with the size of the decision-making body, large or small ${ }^{8}$, that determines the marginal productivity of investment in reputation, viz., the incentive to invest in reputation.

${ }^{8}$ A candidate may be applying for a job at a large corporation or conversely at a small start-up company. Academic program candidates may be facing a large university or a small college. Entrepreneurs can apply for a loan either at a large bank or at a small bank. Assuming that the size of the decision making committee is correlated (positively or negatively) with the size of the organization, 


\section{References}

Bar-Isaac H., and Deb. J., 2014. "What is a good reputation? Career concerns with heterogeneous audiences", International Journal of Industrial Organization 34, 44-50

Ben-Yashar, R. and Danziger. L., 2011. "On the optimal allocation of committee members“, Journal of Mathematical Economics, 47,440-447.

Ben-Yashsr, R., Krausz M. and Nitzan S., 2017. "Government loan guarantees and the credit decision-making structure", Canadian Journal of Economics, forthcoming.

Ben-Yashar, R. and Nitzan, S. 2014. "On the significance of the prior of a correct decision in committees". Theory and Decision, 76, 317-327.

Ben-Yashar, R., and Nitzan, S., 2001. "The Robustness of Optimal Organizational Architectures: A Note on Hierarchies and Polyarchies", Social Choice and Welfare 18, 155-163.

Ben-Yashar, R. and Nitzan, S., 1997. "The optimal decision rule for fixed-size committees in dichotomous choice situations: The general result", International Economic Review 38, 175-186.

Berend, D., \& Paroush, J. 1998. "When is Condorcet's Jury Theorem valid?" Social Choice and Welfare, 15, 481-488.

Bolton G. E. ,A. Ockenfels, F. Ebeling., 2011 "Information value and externalities in reputation building", International Journal of Industrial Organization 29, 23-3.

Condorcet, N.C. de. 1785. "Essai sur l'Application de l'Analyse à la Probabilité des Décisions Rendues à la Pluralité des Voix", Paris. See I. McLean and F. Hewitt, translators, 1994.

Dietrich, F. and List, C. 2013. "Proposition-wise judgment aggregation: The general case", Social Choice and Welfare 40, 1067-1095.

Gerardi, D., Yariv L., 2008. "Information acquisition in committees" Games and Economic Behavior, 62, 436-459.

Grofman, B., Owen, G., Feld, S.L., 1983. "Thirteen theorems in search of the truth". Theory and Decision 15, 261-278.

which may not always be the case, yields clear cut implications regarding the dependence of the incentive to invest in reputation on the size of the organization. 
Kreps D. M., and Wilson R., 1982. "Reputation and Imperfect Information" Journal of Economic Theory 27. 253-279.

Levy, G., 2007. " Decision Making in Committees: Transparency, Reputation, and Voting Rules", The American Economic Review Vol. 97 No. 1, 150-168.

Midjord R., Barraquerb T.R., Valasekc J., 2017. "Voting in large committees with disesteem payoffs: A 'state of the art' model", Games and Economic Behavior 104 $430-443$.

Nitzan, S. and Paroush, J., 1982. "Optimal decision rules in uncertain dichotomous choice situation," International Economic Review 23, 289-297.

Persico, N., 2004, "Committee Design with Endogenous Information", Review of Economic Studies, 71, 165-191.

Sah, R.K. and J. Stiglitz ,1986. "The architecture of economic systems: Hierarchies and polyarchies", American Economic Review, 76, 716-727.

Shapley, L. and Grofman, B., 1984. "Optimizing group judgmental accuracy in the presence of interdependencies," Public Choice 43, 329-343. 


\section{$\underline{\text { Appendix } A}$}

We show that the effect of a change in the a-priory probability of innocence on the probability to acquit a defendant is maximal at the simple majority rule and is minimal at the unanimity rule. ${ }^{9}$

$$
\begin{aligned}
& \frac{\partial \operatorname{Pr}(1: k+1)}{\partial \alpha}>\frac{\partial \operatorname{Pr}(1: k+1+i)}{\partial \alpha}=\frac{\partial \operatorname{Pr}(1: k+1-i)}{\partial \alpha} \text { and } \\
& \frac{\partial\left(\frac{\partial \operatorname{Pr}(1: k+1+i)}{\partial \alpha}\right)}{\partial i}<0 \text {, where } i \text { is a positive integer. }
\end{aligned}
$$

\section{Proof:}

Recall that $\frac{\partial \operatorname{Pr}(1: q)}{\partial \alpha}=T(1: 1)-T(1:-1)=\sum_{j=q}^{n} \Delta_{j}$

where $\Delta_{j}=\left(\begin{array}{c}n \\ j\end{array}\right)\left\{p^{j}(1-p)^{n-j}-(1-p)^{j} p^{n-j}\right\}$.

and

(a) $\forall j>\frac{n}{2}, \Delta_{j}>0$.

(b) $\forall j=a, \Delta_{a}=-\Delta_{n-a}$.

By (a) $\sum_{j=k+1}^{n} \Delta_{j}>\sum_{j=k+1+i}^{n} \Delta_{j}$, hence,

$\frac{\partial \operatorname{Pr}(1: k+1)}{\partial \alpha}>\frac{\partial \operatorname{Pr}(1: k+1+i)}{\partial \alpha}$, where $i$ is a positive integer.

${ }^{9}$ The proof is based on part of the proof of Theorem 2 in Ben-Yashar, Krausz and Nitzan (2017). Their paper focuses on a banking model in which the government uses a guarantee as an instrument for credit inducement and for affecting the bank's decision-making system i.e., its degree of centralization, bias towards approval of loans and reliance on objective loan-specific information. 
By (b) above, if $q<\frac{n}{2}$ then $\sum_{j=q}^{n} \Delta_{j}=\sum_{j=n-q+1}^{n} \Delta_{j}$. Therefore,

$\frac{\partial \operatorname{Pr}(1: q)}{\partial \alpha}=\frac{\partial \operatorname{Pr}(1: n-q+1)}{\partial \alpha}$.

Specifically, this is true when $q=k+1-i$. Substituting $n=2 k+1$ yields, $n-(k+1-i)+1=k+1+i$.

Hence, $\frac{\partial \operatorname{Pr}(1: k+1-i)}{\partial \alpha}=\frac{\partial \operatorname{Pr}(1: k+1+i)}{\partial \alpha}$. Furthermore, $\sum_{j=k+1+i}^{n} \Delta_{j}$ decreases with $i$.

Therefore, $\frac{\partial\left(\frac{\partial \operatorname{Pr}(1: k+1+i)}{\partial \alpha}\right)}{\partial i}<0$.

Q.E.D. 